\title{
How does the hippocampal formation mediate memory for stimuli processed by the magnocellular and parvocellular visual pathways? Evidence from the comparison of schizophrenia and amnestic mild cognitive impairment (aMCI)
}

\author{
Szabolcs Kéri ${ }^{\mathrm{a}, \mathrm{b}, *}$, András Szamosi $^{\mathrm{a}}{ }^{\text {, György Benedek }}{ }^{\mathrm{b}}$, Oguz Kelemen ${ }^{\mathrm{c}}$ \\ a National Psychiatry Center, Budapest, Hungary \\ ${ }^{\mathrm{b}}$ University of Szeged, Faculty of Medicine, Department of Physiology, Dom sq 10, Szeged, Hungary \\ c Bács-Kiskun County Hospital, Psychiatry Center, Kecskemét, Hungary
}

\section{A R T I C L E I N F O}

\section{Article history:}

Received 5 August 2012

Received in revised form

22 September 2012

Accepted 11 October 2012

Available online 22 October 2012

Keywords:

Schizophrenia

Amnestic mild cognitive impairment

Hippocampus

Magnocellular pathway

Vision

Memory

Paired associates learning

\begin{abstract}
A B S T R A C T
Paired associates learning is impaired in both schizophrenia and amnestic mild cognitive impairment (aMCI), which may reflect hippocampal pathology. In addition, schizophrenia is characterized by the dysfunction of the retino-geniculo-striatal magnocellular (M) visual pathway. The purpose of this study was to investigate the interaction between visual perceptual and memory dysfunctions. We administered a modified version of the CANTAB paired associates learning task to patients with schizophrenia $(n=20)$, aMCI $(n=20)$, and two groups of matched healthy controls ( $n=20$ for each patient group). The stimuli in the paired associates learning task biased information processing toward the M pathways (low contrast, low spatial frequency) and parvocellular (P) pathways (high contrast, high spatial frequency). Results revealed that patients with schizophrenia exhibited a more pronounced learning deficit for M-biased relative to P-biased stimuli. In aMCI, there were similar memory deficits for both types of stimuli. Orientation discrimination for $\mathrm{M}$ - and P-biased stimuli was intact in both groups of patients. The number of errors in the M-biased memory condition significantly and inversely correlated with the volume of the right hippocampus in schizophrenia. These results suggest an interaction between

M-biased perceptual processing and short-term relational memory in schizophrenia, which may be associated with the structural alteration of the right hippocampus.
\end{abstract}

(c) 2012 Elsevier Ltd. All rights reserved.

\section{Introduction}

Amnestic mild cognitive impairment (aMCI), a potential prodromal form of Alzheimer's disease, and schizophrenia are fundamentally different disorders regarding etiology and pathogenesis, but both are characterized by a significant impairment of declarative memory (Petersen et al., 1999; Reichenberg \& Harvey, 2007). Although deficits in attention and executive functions contribute to memory dysfunctions, there is ample evidence that pathological processes affect the medial temporal lobe (MTL), including the hippocampus, in both aMCI (Ferreira, Diniz, Forlenza, Busatto, \& Zanetti, 2011; Nickl-Jockschat et al., 2012) and schizophrenia (Heckers \& Konradi, 2010; Stone \& His, 2011; Tamminga, Stan, \& Wagner, 2010).

\footnotetext{
* Corresponding author at: University of Szeged, Faculty of Medicine Department of Physiology, Dom sq 10, Szeged, Hungary. Tel.: +36 204483530 .

E-mail addresses: szkeri2000@yahoo.com, keri.szabolcs.gyula@med.u-szeged.hu (S. Kéri).
}

However, in schizophrenia there is an additional impairment of early-stage visual processes related to the magnocellular (M) pathways, which originate in the retina and project to the primary visual cortex via the M-layers of the lateral geniculate nucleus (reviewed by Javitt, 2009). The adequate stimuli for $\mathrm{M}$ pathways have low luminance contrast and low spatial frequency (coarse resolution of objects). In contrast, the parallel parvocellular $(\mathrm{P})$ pathways prefer static stimuli with high contrast, high spatial frequency (fine details of objects), and colors (Callaway, 2005; Nassi \& Callaway, 2009). M and P pathways interact in primary visual cortex (Sincich \& Horton, 2005), but M pathways provide a definitive input to cortical areas responsible for motion perception, spatial vision, and visuo-motor coordination (dorsal occipito-parietal "stream"). P pathways project to the ventral occipito-temporal region, which serves color perception and object recognition (Van Essen \& Gallant, 1994). However, these higher-level visual cortical areas are not strictly organized in two hierarchical "streams", and a "patchwork" or network model may be more appropriate (de Haan \& Cowey, 2011). In aMCI, early-stage 
vision is spared, but there is a discrete dysfunction of motion- and spatial information-related areas (Lemos, Figueiredo, Santana, Simões, \& Castelo-Branco, 2012; for a review of visuospatial processes in $\mathrm{MCI}$ and Alzheimer's disease, see Iachini, Iavarone, Senese, Ruotolo, \& Ruggiero, 2009).

According to an alternative view of the MTL, the hippocampus cannot be fully understood as a brain region for memory; it also has a fundamental role in perception as an extension of the ventral visual system (Baxter, 2009; Bussey \& Saksida, 2007; Saksida \& Bussey, 2010; for a critical review, see Suzuki, 2009). Lee, Yeung, and Barense (2012) argued that the hippocampus plays a role the visual discrimination of complex spatial scenes, which is beyond the classic role of long-term declarative memory; instead, this function of the hippocampus is reminiscent of that of the dorsal visual system.

Despite these data, it has not been explored how low-level visual features of stimuli interact with MTL functions, such as learning of object-location associations. Recent results indicate that the hippocampus is critical for rapid associative learning with and without consciousness, and memory related to these associations may be both short- and long-term (Henke, 2010). Specifically, we were interested in paired associates learning of stimuli biasing information processing toward the $\mathrm{M}$ and $P$ pathways. Paired associates learning, during which the location of certain stimuli must be acquired, is a sensitive marker of MTL functions (Atienza et al., 2011; Blackwell et al., 2004; de Rover et al., 2011; Hanseeuw et al., 2011; Sahakian et al., 1988; Talpos, Winters, Dias, Saksida, \& Bussey, 2009). We hypothesized that in patients with schizophrenia the impairment of the MTL and M pathways will interact, and patients will show particular difficulties in the learning of paired associates when stimuli are biased toward the M pathways. In contrast, patients with aMCI, who will also be impaired on the paired associates learning task, exhibit similar deficits on tasks using $\mathrm{M}$ - and P-biased stimuli. In our previous study, we found impaired paired associates learning in aMCI, and smaller right, but not left, hippocampal volume was associated with more errors on the task (Levy-Gigi, Kelemen, Gluck, \& Kéri, 2011). However, in the study of Levy-Gigi et al. (2011) stimuli were not biased toward specific visual pathways. Based on these data and studies reporting the role of hippocampus in spatial vision (Lee et al., 2012), we hypothesized a correlation between paired associates learning for M-biased stimuli and right hippocampal volume.

\section{Method}

\subsection{Participants}

Twenty patients with schizophrenia, 20 patients with aMCI, and two age-, education-, and gender-matched healthy control groups participated in the study (Table 1). The diagnosis of schizophrenia was based on structured clinical interviews (First, Spitzer, Gibbon, \& Williams, 1996) and medical records. The patients with schizophrenia received antipsychotic medications at the time of testing (risperidone, $n=7$; olanzapine, $n=7$; quetiapine, $n=4$; haloperidol, $n=2$; mean chlorpromazine-equivalent dose: $430.5 \mathrm{mg} /$ day, $S D=160.4$ ) (Woods, 2003).

The severity of the symptoms was characterized by the Brief Psychiatric Rating Scale (Overall \& Gorham, 1962) (mean: 41.6, $S D=7.3$ ).

For the diagnosis of aMCI, we used the Mayo Clinic Alzheimer's Disease Research Center criteria (Petersen et al., 1999). Exclusion criteria included history of neurological or psychiatric disorders, head trauma, substance misuse, and medications affecting central nervous system functions. None of patients with aMCI received psychotropic medications at the time of testing. The mean MiniMental State Examination (Folstein, Folstein, \& McHugh, 1975) score was 26.2 $(S D=1.2)$ in the aMCI group.

\subsection{Stimulus presentation}

We used a VP2765-LED-27" monitor (ViewSonic, Walnut, CA; refresh rate: $60 \mathrm{~Hz}$, resolution: $1920 \times 1080$ pixel; viewing distance: $50 \mathrm{~cm}$; output luminance: $65 \mathrm{~cd} / \mathrm{m}^{2}$; size: $28^{\circ}$ ) controlled by a Dell XPS workstation. All experiments ran in a MATLAB environment (MathWorks, Natick).

2.3. Paired associates learning with stimuli biasing information processing toward the $M$ and $P$ pathways

The procedure was based on the Cambridge Neuropsychological Test Automated Battery (CANTAB) paired associates learning test (Sahakian et al., 1988). We used the short version of the test during which 3 or 6 visual patterns were displayed in boxes placed in 6 different locations on the computer screen. The pattern was then presented in the center of the display, and the task was to click on the box in which the pattern had been seen. There were 10 presentations and recall phases if participants had not localized all patterns correctly $(5 \times 3$ and $5 \times 6$ effective trials in the 3- and 6-pattern task, respectively). In the 6-pattern task none of the participants was able to recall all items in a display without errors, and therefore the number of total trials was the same in each participant. The 3-item task, in which active short-term rehearsal of items is more likely than in the 6-pattern task, served as a warm-up procedure to familiarize the participants with general task requirements. The dependent measure was the number of errors in the 6-pattern task.

In contrast to CANTAB, in which patterns vary in shape and color, we used Gabor patches with different contrast, spatial frequency, and orientation. Gabor patches were generated as described in our previous study (Kéri, Kiss, Kelemen, Benedek, \& Janka, 2005). The stimuli are illustrated in Fig. 1. In the M pathway condition, the spatial frequency of the stimuli was 1 cycle $/{ }^{\circ}$, and the Michelsoncontras was $8 \%$. Before the experiment, we tested all participants to ensure that they were able to detect this contrast level. In the P pathway condition, the spatial

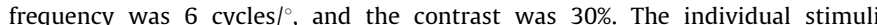
presented in separate boxes differed in orientation $\left(0-180^{\circ}\right.$, differences of 30 across stimuli) and subtended $5 \times 5^{\circ}$ of visual angle. The exposure time was $100 \mathrm{~ms}$. There were two counterbalanced blocks including $\mathrm{M}$ - and P-biased stimuli.

Low spatial frequency

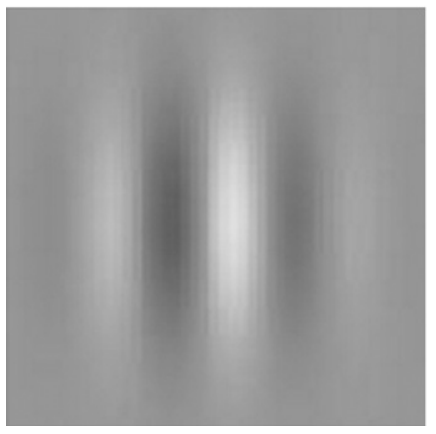

Fig. 1. Illustration of the Gabor patches with low and high spatial frequency

Table 1

Characteristics of the participants.

\begin{tabular}{llcc}
\hline & Schizophrenia-controls & Schizophrenia-patients & aMCI-controls \\
\hline Age (years) & $38.6(5.4)$ & $39.0(6.3)$ & $65.4(4.1)$ \\
Gender (male/female) & $12 / 8$ & $12 / 8$ & $7 / 13$ \\
Education (years) & $11.6(2.9)$ & $11.2(3.5)$ & $13.5(3.6)$ \\
Time since diagnosis (months) & - & $183.5(61.3)$ & - \\
\hline
\end{tabular}

Data are mean (standard deviation). Control and patient groups were matched for age, education, and gender $(p>0.5)$. 


\subsection{Perceptual control task: Orientation discrimination}

We used the Gabor patches from the paired associate learning experiment. The exposure time was $100 \mathrm{~ms}$, similarly to the paired associates learning test. The task was to decide whether the stimulus was closer to the horizontal or vertical orientation by pressing two different keys. There were 10 trials for M-biased stimuli and 10 trials for P-biased stimuli. The dependent measure was the number of errors.

\subsection{Structural brain imaging}

We followed the FreeSurfer procedure for optimal volumetric measurements (Martinos Center for Biomedical Imaging, Boston, MA, USA; http://surfer.nmr.mgh. harvard.edu; version: v5.1.0, Dell XPS workstation). A multiecho FLASH sequence was used with a $1 \mathrm{~mm}^{3}$ isotropic resolution (Siemens Trio 3T scanner; $256 \times 256$ matrix, 176 sagittal slices with a thickness of $1 \mathrm{~mm}$, TR $2530 \mathrm{~ms}$, TI $1100 \mathrm{~ms}$, TE $1.64 / 3.5 / 5.36 / 7.22 \mathrm{~ms}$, bandwidth $651 \mathrm{~Hz}$, non-selective excitation at $7^{\circ}$ ). Image processing consisted of the following steps: removal of non-brain tissue with a hybrid watershed/surface deformation technique, automated Talairach transformation, and segmentation of white and grey matter (Fischl et al., 2004; Segonne et al., 2004; for methodological limitations, see Gronenschild et al., 2012). We measured the volume of the left and right hippocampus normalized to the intracranial volume.

\subsection{Statistical analysis}

First, using STATISTICA 9 software (StatSoft Inc., Tulsa), we ran KolmogorovSmirnov tests to assess the normality of data distribution before the application of parametric tests. Demographic variables and hippocampal volumes were compared with two-tailed $t$ tests. Repeated-measures analyses of variance (ANOVAs) were conducted to compare orientation discrimination and paired associates learning performance, followed by Scheffé's post hoc tests. We calculated Pearson's product moment correlation coefficients between test performance and hippocampal volume. The level of statistical significance was $\alpha<0.05$.

\section{Results}

\subsection{Orientation discrimination}

The results are in Table 2 . We conducted a group (schizophrenia vs. controls) by stimulus type (M- and P-biased) ANOVA. This ANOVA revealed no significant main effects or a two-way interaction $(F<2.5, p>0.1)$. In the aMCI vs. control comparison, there were no significant differences, too $(F<1, p>0.5)$ (Table 2$)$.

\subsection{Paired associates learning}

In the schizophrenia vs. controls comparison, there were significant main effects of group $(F(1,38)=63.04, p<0.001$, $\left.\eta^{2}=0.62\right)$ and stimulus type $(F(1,38)=21.92, \quad p<0.001$, $\left.\eta^{2}=0.37\right)$. The interaction between group and stimulus type was

Table 2

Results from the orientation discrimination and paired associates tasks.

\begin{tabular}{lllll}
\hline & $\begin{array}{l}\text { Schizophrenia } \\
\text { controls }(n=20)\end{array}$ & $\begin{array}{l}\text { Schizophrenia } \\
\text { patients }(n=20)\end{array}$ & $\begin{array}{l}\text { aMCI } \\
\text { controls } \\
(n=20)\end{array}$ & $\begin{array}{l}\text { aMCI } \\
\text { patients } \\
(n=20)\end{array}$ \\
\hline $\begin{array}{l}\text { Orientation discrimination } \\
\text { Magnocellular }\end{array}$ & $1.3(0.8)$ & $1.6(1.0)$ & $1.7(1.0)$ & $1.9(1.4)$ \\
$\quad \begin{array}{l}\text { Parvocellular } \\
1.3(0.9)\end{array}$ & $1.8(1.1)$ & $1.8(1.4)$ & $2.0(1.1)$ \\
$\begin{array}{l}\text { Paired associates learning } \\
\text { Magnocellular }\end{array} 6.3(3.7)$ & $18.9(4.8)$ & $7.7(3.2)$ & $14.6(4.1)$ \\
Parvocellular & $7.2(4.1)$ & $11.5(3.3)$ & $8.0(3.6)$ & $15.0(5.2)$ \\
\hline
\end{tabular}

Data are mean number of errors (standard deviation). aMCI-amnestic mild cognitive impairment.

${ }^{a}$ No significant between-group differences (matched patients and controls).

b Patients with schizophrenia were more impaired on the magnocellular relative to the parvocellular condition, whereas in aMCI the impairment was equivocal for both stimulus types (for a detailed statistical analysis, see the text). also significant $\left(F(1,38)=35.73, p<0.001, \eta^{2}=0.49\right)$. Scheffé's tests yielded that patients with schizophrenia made significantly more errors than did the control group in both $\mathrm{M}(p<0.001)$ and $\mathrm{P}(p<0.05)$ conditions, although these between-group differences were not equal. The controls performed similarly for $\mathrm{M}$ - and P-biased stimuli $(p=0.8)$, whereas patients with schizophrenia performed less efficiently on the M-biased relative to the P-biased condition $(p<0.001)$ (Table 2$)$.

In the aMCI vs. control comparison, we found a significant main effect of group $\left(F(1,38)=37.73, p<0.001, \eta^{2}=0.47\right)$, but the interaction between stimulus type and group did not reach the level of statistical significance $(F<1, p>0.5)$ (Table 2$)$.

\subsection{Hippocampal volume and correlations with test results}

Table 3 depicts the volumetric data, which indicate bilateral hippocampal volume reduction in both schizophrenia and aMCI. There were several nonsignificant correlations between test performances and hippocampal volume $(-0.3<r<0.3)$ : (i) orientation discrimination in each group with both hippocampi; (ii) paired associates learning in controls with both hippocampi; (iii) left hippocampal volume and paired associates learning in patients. In patients with schizophrenia, right hippocampal volume significantly correlated with paired associates learning for M-biased stimuli (smaller volume-more errors, $r=-0.67, p=0.001)$ but not for P-biased stimuli $(r=-0.18$, $p=0.5$ ) (Fig. 2). These two correlation coefficients were significantly different (one-tailed, $p=0.04$ ). However, this difference did not reach the level of statistical significance when a two-tailed test was used $(p=0.08)$, and performance range was smaller in the P-biased condition. In patients with aMCI, paired associates learning was significantly related to the volume of the right hippocampus for both types of stimuli (M-biased: $r=-0.50$, $p<0.05$; P-biased: $r=-0.53, p<0.05$ ) (Fig. 3).

\subsection{Correlations with antipsychotic dose and symptoms}

There were no significant correlations between test performances, hippocampal volume, daily chlorpromazine-equivalent antipsychotics, BPRS and MMSE scores, and duration of symptoms (time since diagnosis) $(-0.1<r<0.1)$.

\section{Discussion}

In accordance with the hypothesis of the study, we found impaired paired associates learning in both patients with schizophrenia and aMCI, which is broadly consistent with the literature (Armstrong, Kose, Williams, Woolard, \& Heckers, 2012; Atienza et al., 2011; Blackwell et al., 2004; de Rover et al., 2011; Elvevag, Egan, \& Goldberg, 2000; Ongür et al., 2006; Hanseeuw et al., 2011). Reduced hippocampal volume is also a well-known neuroanatomical alteration in these disorders (Adriano, Caltagirone, \& Spalletta, 2012; Ferreira et al., 2011; Nickl-Jockschat et al., 2012; Steen, Mull, McClure, Hamer, \& Lieberman, 2006). However, the impact of physical characteristics of the stimuli (spatial frequency and contrast) influenced memory performance only in patients with schizophrenia, exhibiting more errors for Mbiased low spatial frequency/low contrast Gabor patches relative to P-biased high spatial frequency/high contrast stimuli. In both patient populations, impaired learning was associated with reduced right hippocampal volume, and this correlation was particularly evident in patients with schizophrenia in the case of M-biased stimuli. The prominent role of the right, but not left, hippocampal formation in visual memory for object-location associations is well established (e.g., Braun et al., 2011; Manelis, 
Table 3

Hippocampal volumes $\left(\mathrm{mm}^{3}\right)$

\begin{tabular}{lllll}
\hline & Schizophrenia-controls & Schizophrenia-patients & aMCI-controls & aMCI-patients \\
\hline Left hippocampus & $3873.0(283.2)$ & $3609.1(333.4)$ & $3700.4(220.9)$ & $3459.3(241.9)$ \\
Right hippocampus & $3896.0(276.7)$ & $3589.5(287.4)$ & $3733.2(217.6)$ & $3556.5(240.9)$ \\
\hline
\end{tabular}

Data are mean (standard deviation). aMCI—amnestic mild cognitive impairment, Schizophrenia patients vs. controls: right, $t(38)=2.96, p=0.005$; left, $t(38)=3.14, p=0.003$; aMCI patients vs. controls: right, $t(38)=2.43, p=0.02 ;$ left, $t(38)=3.30, p=0.002$.

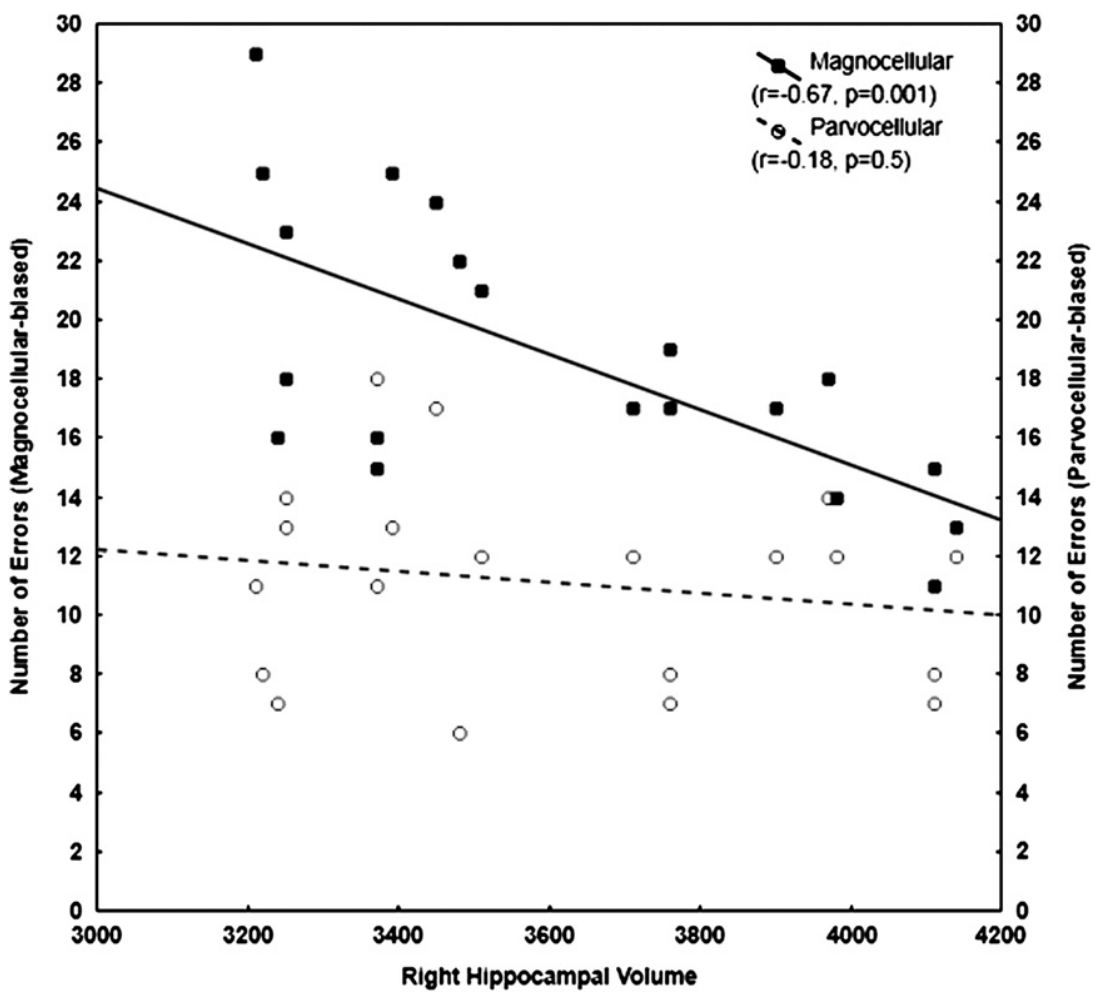

Fig. 2. Correlation between right hippocampal volume $\left(\mathrm{mm}^{3}\right)$ and paired associates learning (number of errors) in patients with schizophrenia.

Reder, \& Hanson, 2012; Piekema, Kessels, Mars, Petersson, \& Fernández, 2006). Interestingly, patients and controls performed similarly on the orientation discrimination task, suggesting a relative specificity of findings for memory processes, that is, the deficit cannot be explained by reduced sensory capacities leading to a failure to differentiate the orientation of the stimuli. Consistent with this finding, Yoon, Seo, Kim, and Lee (2011) demonstrated that pharmacological inactivation of the dorsal hippocampus in animal experiments disrupted paired associates learning, whereas it did not affect the discrimination of individual objects or locations.

Regarding the limitations of the study, the most important question is the feasibility of the selective stimulation of $\mathrm{M}$ and $\mathrm{P}$ pathways. Evidence from animal experiments, human electrophysiological and functional neuroimaging studies suggests that information processing can be biased toward $\mathrm{M}$ and $\mathrm{P}$ pathways by using stimuli with different spatial frequencies and contrasts, although an entirely selective stimulation is not likely in human psychophysical experiments (for review and discussion, see Butler et al., 2007a,b; Skottun \& Skoyles, 2007). Additionally, "magnocellular function" is not a unitary construct, and different psychophysical tasks reflect different sources of variance instead of a general behavioral marker of M pathways (Goodbourn et al., 2012).

The second issue is the question of antipsychotic medication, which can affect visual information processing (Chen et al., 2003;
Kéri, Antal, Szekeres, Benedek, \& Janka, 2002; Kiss, Fábián, Benedek, \& Kéri, 2010). However, this effect seems to be especially pronounced when stimuli at the perceptual threshold are used, i.e., in contrast sensitivity measurements; in the current study there was no significant correlation between antipsychotic dose and test performance. Similarly, the global severity of clinical symptoms did not correlate with test performance, although we did not examine the potentially distinct contributions of positive, negative, and disorganized symptoms (we only had the total BPRS score and the sample size was small).

Third, despite the fact that paired associates learning is a wellestablished paradigm for the assessment of hippocampal functions, and the correlation between task performance and right hippocampal volume was predicted by previous results (Levy-Gigi et al., 2011), the anatomical specificity of our findings is not absolute given that other brain regions (e.g., prefrontal and parietal areas) were not investigated. Finally, in the orientation discrimination test we observed a floor effect in controls, and therefore it should be considered as a perceptual competency task and not a real control condition.

The finding that physical characteristics of stimuli may interfere with the functions of brain structures related to higher-level cognition in schizophrenia is consistent with the functional neuroimaging literature. For example, Martínez et al. (2008) showed that, relative to healthy controls, patients with schizophrenia exhibited reduced activations to low, but not high, spatial 


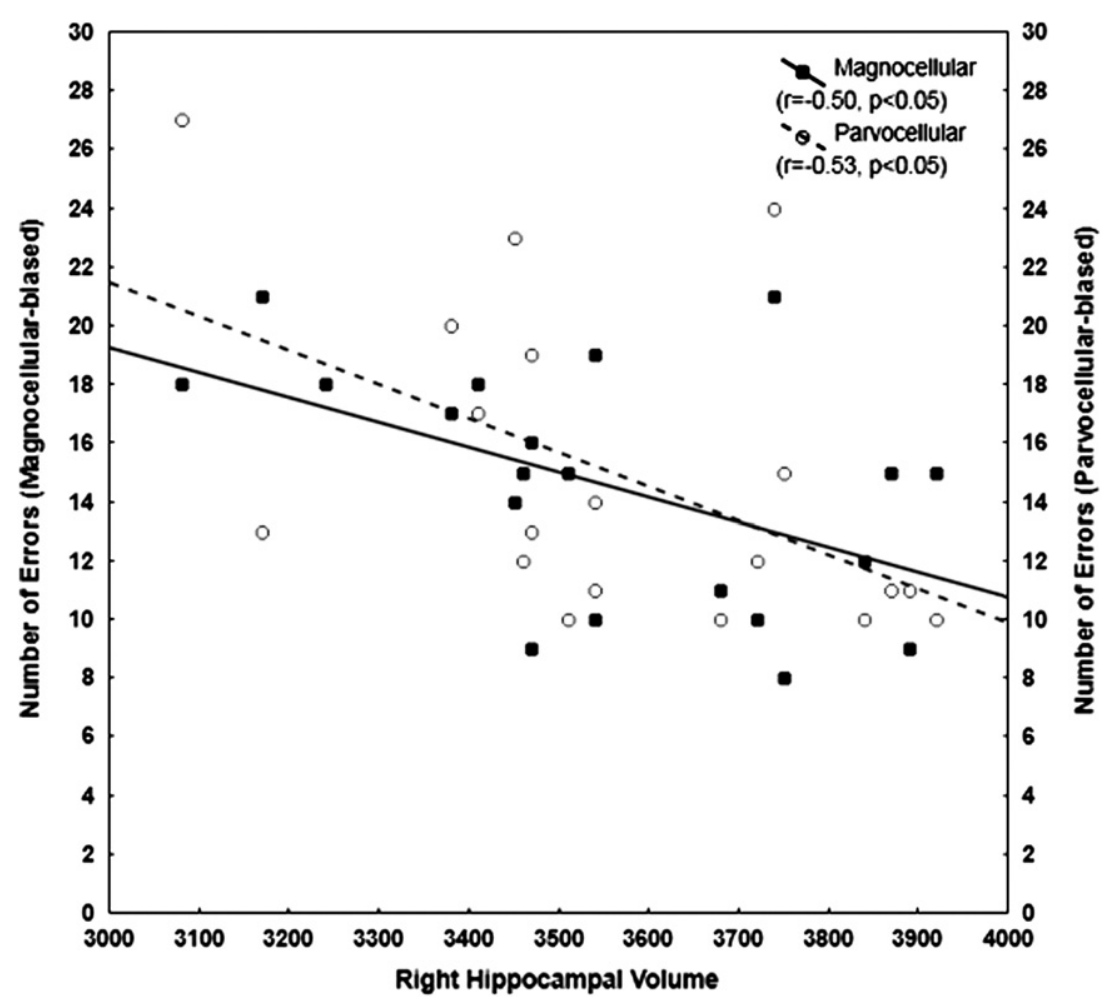

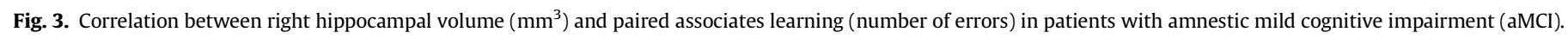

frequencies in the occipital, parietal, and temporal cortical areas (but see Laprévote, Oliva, Delerue, Thomas, \& Boucart, 2010). Using a combined event-related potential and functional magnetic resonance imaging approach in patients with schizophrenia, Sehatpour et al. (2010) demonstrated impaired early-stage sensory processing and deficient activation of the dorsal and ventral visual regions, prefrontal cortex, and hippocampus. Structural equation modeling revealed that impaired activation of dorsal visual regions contributed to impaired prefrontal activation, which in turn contributed significantly to impaired activation of the hippocampus and ventral visual regions (Sehatpour et al., 2010). These results from electrophysiological and functional neuroimaging studies are supported and extended by the findings of the present study, which used anatomical volumetric measurements of the hippocampal formation and neuropsychological comparisons with aMCI.

It is necessary to emphasize that hippocampal atrophy in general did not result in differentially impaired learning of M-biased stimuli. Patients with aMCI were equally impaired on tests using M- and P-biased Gabor patches, despite the fact that they displayed a seemingly similar hippocampal volume loss compared with that of patients with schizophrenia. In aMCI, errors on the paired associates learning test correlated with smaller hippocampal volume for both $\mathrm{M}$ - and P-biased stimuli, whereas this correlation was selective for M-biased stimuli in schizophrenia. Two issues must be taken into consideration in the interpretation of these results. First, evidence indicates that the processing of M-biased stimuli is disrupted in schizophrenia even before the primary visual cortex (Butler et al., 2005), and therefore higher-level visual areas and the hippocampal formation will receive degraded information. There is no evidence for a similar dysfunction in aMCI. Second, the MTL, including the hippocampus, consists of morphologically and functionally distinct modules, which might not be impaired in the same way in schizophrenia and aMCI. For example, Mueller et al. (2010) demonstrated that the atrophy of the CA1-2 zone is characteristic for patients with aMCI, whereas in schizophrenia CA1 and CA2/3 subfields may be differentially affected (Schobel et al., 2009; Kühn et al., 2012). Zone CA1 may play a distinctive role in memory encoding (Fouquet et al., 2012), whereas zone CA3 may be implicated in spatial processing (Kesner \& Hopkins, 2006). Thome, Erickson, Lipa, and Barnes (2012) found visual image-selective neurons in the CA3 region of the macaque monkey that did not alter their activity when the image became familiar, suggesting their dominant role in visual perception. However, human MTL subfield analysis is in an early stage and therefore a special care is needed in the interpretation of these results (Small, Schobel, Buxton, Witter, \& Barnes, 2011; discussion by van Strien, Widerøe, van de Berg, \& Uylings, 2011). In addition, it may be an oversimplification to claim that specific clinical symptoms or sensory and cognitive functions can be mapped onto a single brain region in a mechanistic manner.

In conclusion, we demonstrated an intriguing interaction between perception and short-term relational memory in schizophrenia: the formation of stimulus-location associations was less efficient in the case of M-biased relative to P-biased stimuli, which was significantly related to smaller right hippocampal volume. We did not observe this differential deficit in aMCI where both $\mathrm{M}$ - and P-biased stimuli were similarly affected, and the relationship with hippocampal volume was weaker. We clearly need future studies to elucidate the functional activation of the MTL in schizophrenia during the encoding and retrieval of M- vs. P-biased stimuli, as well as basic research investigating the relationship between $\mathrm{M}$ and $\mathrm{P}$ pathways, visual cortical areas, and the MTL. These studies may highlight the enduring question of separation vs. continuity between low-level perception and higher-level cognition (e.g., Bar, 2003; Lages \& Paul, 2006; Magnussen, Greenlee, Aslaksen, \& Kildebo, 2003; Singh, Stojanoski, Le, \& Niemeier, 2011; Sowden \& Schyns, 2006). 


\section{Acknowledgments}

This study was supported by the Hungarian Research Fund (OTKA NF72488).

\section{References}

Adriano, F., Caltagirone, C., \& Spalletta, G. (2012). Hippocampal volume reduction in first-episode and chronic schizophrenia: a review and meta-analysis. Neuroscientist, 18, 180-200.

Armstrong, K., Kose, S., Williams, L., Woolard, A., \& Heckers, S. (2012). Impaired associative inference in patients with schizophrenia. Schizophrenia Bulletin, 38, 622-629.

Atienza, M., Atalaia-Silva, K. C., Gonzalez-Escamilla, G., Gil-Neciga, E., SuarezGonzalez, A., \& Cantero, J. L. (2011). Associative memory deficits in mild cognitive impairment: the role of hippocampal formation. Neuroimage, 57, 1331-1342.

Bar, M. (2003). A cortical mechanism for triggering top-down facilitation in visual object recognition. Journal of Cognitive Neuroscience, 15, 600-609.

Baxter, M. G. (2009). Involvement of medial temporal lobe structures in memory and perception. Neuron, 61, 667-677.

Blackwell, A. D., Sahakian, B. J., Vesey, R., Semple, J. M., Robbins, T. W., \& Hodges, J. R. (2004). Detecting dementia: novel neuropsychological markers of preclinical Alzheimer's disease. Dementia and Geriatric Cognitive Disorders, 17, 42-48.

Braun, M., Weinrich, C., Finke, C., Ostendorf, F., Lehmann, T. N., \& Ploner, C. J. (2011). Lesions affecting the right hippocampal formation differentially impair short-term memory of spatial and nonspatial associations. Hippocampus, 21, 309-318.

Bussey, T. J., \& Saksida, L. M. (2007). Memory, perception, and the ventral visualperirhinal-hippocampal stream: thinking outside of the boxes. Hippocampus, 17, 898-908.

Butler, P. D., Martinez, A., Foxe, J. J., Kim, D., Zemon, V., Silipo, G., et al. (2007a). Subcortical visual dysfunction in schizophrenia drives secondary cortical impairments. Brain, 130, 417-430.

Butler, P. D., Martinez, A., Foxe, J. J., Kim, D., Zemon, V., Silipo, G., et al. (2007b) Reply: a few remarks on assessing magnocellular sensitivity in schizophrenic patients. Brain, 130, e84.

Butler, P. D., Zemon, V., Schechter, I., Saperstein, A. M., Hoptman, M. J., Lim, K. O., et al. (2005). Early-stage visual processing and cortical amplification deficits in schizophrenia. Archives of General Psychiatry, 62, 495-504.

Callaway, E. M. (2005). Structure and function of parallel pathways in the primate early visual system. Journal of Physiology, 566, 13-19.

Chen, Y., Levy, D. L., Sheremata, S., Nakayama, K., Matthysse, S., \& Holzman, P. S. (2003). Effects of typical, atypical, and no antipsychotic drugs on visual contrast detection in schizophrenia. American Journal of Psychiatry, 160, 1795-1801.

de Haan, E. H., \& Cowey, A. (2011). On the usefulness of 'what' and 'where' pathways in vision. Trends in Cognitive Sciences, 15, 460-466.

de Rover, M., Pironti, V. A., McCabe, J. A., Acosta-Cabronero, J., Arana, F. S., MoreinZamir, S., et al. (2011). Hippocampal dysfunction in patients with mild cognitive impairment: a functional neuroimaging study of a visuospatial paired associates learning task. Neuropsychologia, 49, 2060-2070.

Elvevag, B., Egan, M. F., \& Goldberg, T. E. (2000). Paired-associate learning and memory interference in schizophrenia. Neuropsychologia, 38, 1565-1575.

Ferreira, L. K., Diniz, B. S., Forlenza, O. V., Busatto, G. F., \& Zanetti, M. V. (2011). Neurostructural predictors of Alzheimer's disease: a meta-analysis of VBM studies. Neurobiology of Aging, 32, 1733-1741.

First, M. B., Spitzer, R. L., Gibbon, M., \& Williams, J. B. W. (1996). Structured clinical interview for DSM-IV axis I disorders, clinician version (SCID-CV). Washington, DC: American Psychiatric Press.

Fischl, B., Salat, D. H., van der Kouwe, A. J., Makris, N., Ségonne, F., Quinn, B. T., et al. (2004). Sequence-independent segmentation of magnetic resonance images. Neuroimage, 23(1), S69-S84

Folstein, M. F., Folstein, S. E., \& McHugh, P. R. (1975). Mini-mental state. A practical method for grading the cognitive state of patients for the clinician. Journal of Psychiatric Research, 12, 189-198.

Fouquet, M., Desgranges, B., La Joie, R., Rivière, D., Mangin, J. F., Landeau, B., et al. (2012). Role of hippocampal CA1 atrophy in memory encoding deficits in amnestic mild cognitive impairment. Neuroimage, 59, 3309-3315.

Goodbourn, P. T., Bosten, J. M., Hogg, R. E., Bargary, G., Lawrance-Owen, A. J., \& Mollon, J. D. (2012). Do different 'magnocellular tasks' probe the same neural substrate? Philosophical Transaction of the Royal Society B: Biological Sciences, 279, 4263-4271.

Gronenschild, E. H., Habets, P., Jacobs, H. I., Mengelers, R., Rozendaal, N., van Os, J., et al. (2012). The effects of FreeSurfer version, workstation type, and Macintosh operating system version on anatomical volume and cortical thickness measurements. PLoS One, 7, e38234.

Hanseeuw, B., Dricot, L., Kavec, M., Grandin, C., Seron, X., \& Ivanoiu, A. (2011). Associative encoding deficits in amnestic mild cognitive impairment: a volumetric and functional MRI study. Neuroimage, 56, 1743-1748.

Heckers, S., \& Konradi, C. (2010). Hippocampal pathology in schizophrenia. Current Topics in Behavioral Neuroscience, 4, 529-553.
Henke, K. (2010). A model for memory systems based on processing modes rather than consciousness. Nature Reviews Neuroscience, 11, 523-532.

Iachini, I., Iavarone, A., Senese, V. P., Ruotolo, F., \& Ruggiero, G. (2009). Visuospatial memory in healthy elderly, $\mathrm{AD}$ and $\mathrm{MCl}$ : a review. Current Aging Science, 2, 43-59.

Javitt, D. C. (2009). When doors of perception close: bottom-up models of disrupted cognition in schizophrenia. Annual Review of Clinical Psychology, 5, 249-275.

Kéri, S., Antal, A., Szekeres, G., Benedek, G., \& Janka, Z. (2002). Spatiotemporal visual processing in schizophrenia. Journal of Neuropsychiatry and Clinical Neurosciences, 14, 190-196.

Kéri, S., Kiss, I., Kelemen, O., Benedek, G., \& Janka, Z. (2005). Anomalous visual experiences, negative symptoms, perceptual organization and the magnocellular pathway in schizophrenia: a shared construct? Psychological Medicine, 35 1445-1455.

Kesner, R. P., \& Hopkins, R. O. (2006). Mnemonic functions of the hippocampus: comparison between animals and humans. Biological Psychology, 73, 3-18.

Kiss, I., Fábián, A., Benedek, G., \& Kéri, S. (2010). When doors of perception open: visual contrast sensitivity in never-medicated, first-episode schizophrenia. Journal of Abnormal Psychology, 119, 586-593.

Kühn, S., Musso, F., Mobascher, A., Warbrick, T., Winterer, G., \& Gallinat, J. (2012) Hippocampal subfields predict positive symptoms in schizophrenia: first evidence from brain morphometry. Translational Psychiatry, 2, e127.

Lages, M., \& Paul, A. (2006). Visual long-term memory for spatial frequency? Psychonomic Bulletin \& Review, 13, 486-492.

Laprévote, V., Oliva, A., Delerue, C., Thomas, P., \& Boucart, M. (2010). Patients with schizophrenia are biased toward low spatial frequency to decode facial expression at a glance. Neuropsychologia, 48, 4164-4168.

Lee, A. C., Yeung, L. K., \& Barense, M. D. (2012). The hippocampus and visual perception. Frontiers in Human Neuroscience, 6, 91.

Lemos, R., Figueiredo, P., Santana, I., Simões, M. R., \& Castelo-Branco, M. (2012) Temporal integration of 3D coherent motion cues defining visual objects of unknown orientation is impaired in amnestic mild cognitive impairment and Alzheimer's disease. Journal of Alzheimer's Disease, 28, 885-896.

Levy-Gigi, E., Kelemen, O., Gluck, M. A., \& Kéri, S. (2011). Impaired context reversa learning, but not cue reversal learning, in patients with amnestic mild cognitive impairment. Neuropsychologia, 49, 3320-3326.

Magnussen, S., Greenlee, M. W., Aslaksen, P. M., \& Kildebo, O. O. (2003). Highfidelity perceptual long-term memory revisited-and confirmed. Psychological Science, 14, 74-76.

Manelis, A., Reder, L. M., \& Hanson, S. J. (2012). Dynamic changes in the medial temporal lobe during incidental learning of object-location associations. Cerebral Cortex, 22, 828-837.

Martínez, A., Hillyard, S. A., Dias, E. C., Hagler, D. J., Jr, Butler, P. D., Guilfoyle, D. N et al. (2008). Magnocellular pathway impairment in schizophrenia: evidence from functional magnetic resonance imaging. Journal of Neuroscience, 28 7492-7500.

Mueller, S. G., Schuff, N., Yaffe, K., Madison, C., Miller, B., \& Weiner, M. W. (2010) Hippocampal atrophy patterns in mild cognitive impairment and Alzheimer's disease. Human Brain Mapping, 31, 1339-1347.

Nassi, J. J., \& Callaway, E. M. (2009). Parallel processing strategies of the primate visual system. Nature Reviews Neuroscience, 10, 360-372.

Nickl-Jockschat, T., Kleiman, A., Schulz, J. B., Schneider, F., Laird, A. R., Fox, P. T. et al. (2012). Neuroanatomic changes and their association with cognitive decline in mild cognitive impairment: a meta-analysis. Brain Structure and Function, 217, 115-125.

Ongür, D., Cullen, T. J., Wolf, D. H., Rohan, M., Barreira, P., Zalesak, M., et al. (2006) The neural basis of relational memory deficits in schizophrenia. Archives of General Psychiatry, 63, 356-565.

Overall, J. E., \& Gorham, D. R. (1962). The brief psychiatric rating scale. Psychological Reports, 10, 799-812.

Petersen, R. C., Smith, G. E., Waring, S. C., Ivnik, R. J., Tangalos, E. G., \& Kokmen, E. (1999). Mild cognitive impairment: clinical characterization and outcome. Archives of Neurology, 56, 303-308.

Piekema, C., Kessels, R. P., Mars, R. B., Petersson, K. M., \& Fernández, G. (2006). The right hippocampus participates in short-term memory maintenance of objectlocation associations. Neuroimage, 33, 374-382.

Reichenberg, A., \& Harvey, P. D. (2007). Neuropsychological impairments in schizophrenia: integration of performance-based and brain imaging findings. Psychological Bulletin, 133, 833-858.

Sahakian, B. J., Morris, R. G., Evenden, J. L., Heald, A., Levy, R., Philpot, M., et al. (1988). A comparative study of visuospatial memory and learning in Alzheimer-type dementia and Parkinson's disease. Brain, 111, 695-718.

Saksida, L. M., \& Bussey, T. J. (2010). The representational-hierarchical view of amnesia: translation from animal to human. Neuropsychologia, 48, 2370-2384.

Schobel, S. A., Lewandowski, N. M., Corcoran, C. M., Moore, H., Brown, T. Malaspina, D., et al. (2009). Differential targeting of the CA1 subfield of the hippocampal formation by schizophrenia and related psychotic disorders. Archives of General Psychiatry, 66, 938-946.

Segonne, F., Dale, A. M., Busa, E., Glessner, M., Salat, D., Hahn, H. K., et al. (2004) A hybrid approach to the skull stripping problem in MRI. Neuroimage, 22, 1060-1075.

Sehatpour, P., Dias, E. C., Butler, P. D., Revheim, N., Guilfoyle, D. N., Foxe, J. J., et al (2010). Impaired visual object processing across an occipital-frontalhippocampal brain network in schizophrenia: an integrated neuroimaging study. Archives of General Psychiatry, 67, 772-782. 
Sincich, L. C., \& Horton, J. C. (2005). The circuitry of V1 and V2: integration of color, form, and motion. Annual Review of Neuroscience, 28, 303-326.

Singh, V. V., Stojanoski, B., Le, A., \& Niemeier, M. (2011). Spatial frequency-specific effects on the attentional bias: evidence for two attentional systems. Cortex $47,547-556$

Skottun, B. C., \& Skoyles, J. (2007). A few remarks on assessing magnocellular sensitivity in schizophrenic patients. Brain, 130, e83.

Small, S. A., Schobel, S. A., Buxton, R. B., Witter, M. P., \& Barnes, C. A. (2011). A pathophysiological framework of hippocampal dysfunction in ageing and disease. Nature Reviews Neuroscience, 12, 585-601.

Sowden, P. T., \& Schyns, P. G. (2006). Channel surfing in the visual brain. Trends in Cognitive Sciences, 10, 538-545.

Steen, R. G., Mull, C., McClure, R., Hamer, R. M., \& Lieberman, J. A. (2006). Brain volume in first-episode schizophrenia: systematic review and meta-analysis of magnetic resonance imaging studies. British Journal of Psychiatry, 188, 510-518.

Stone, W. S., \& His, X. (2011). Declarative memory deficits and schizophrenia: problems and prospects. Neurobiology of Learning and Memory, 96, 544-552.

Suzuki, W. A. (2009). Perception and the medial temporal lobe: evaluating the current evidence. Neuron, 61, 657-666.
Talpos, J. C., Winters, B. D., Dias, R., Saksida, L. M., \& Bussey, T. J. (2009). A novel touchscreen-automated paired-associate learning (PAL) task sensitive to pharmacological manipulation of the hippocampus: a translational rodent model of cognitive impairments in neurodegenerative disease. Psychopharmacology (Berlin), 205, 157-168.

Tamminga, C. A., Stan, A. D., \& Wagner, A. D. (2010). The hippocampal formation in schizophrenia. American Journal of Psychiatry, 167, 1178-1193.

Thome, A., Erickson, C. A., Lipa, P., \& Barnes, C. A. (2012). Differential effects of experience on tuning properties of macaque MTL neurons in a passive viewing task. Hippocampus, 22, 2000-2111.

Van Essen, D. C., \& Gallant, J. L. (1994). Neural mechanisms of form and motion processing in the primate visual system. Neuron, $13,1-10$.

van Strien, N. M., Widerøe, M., van de Berg, W. D., \& Uylings, H. B. (2011). Imaging hippocampal subregions with in vivo MRI: advances and limitations. Nature Reviews Neuroscience, 13, 70.

Woods, S. W. (2003). Chlorpromazine equivalent doses for the newer atypical antipsychotics. Journal of Clinical Psychiatry, 64, 663-667.

Yoon, J., Seo, Y., Kim, J., \& Lee, I. (2011). Hippocampus is required for paired associate memory with neither delay nor trial uniqueness. Learning and Memory, 19, 1-8. 